\title{
Assistierter Suizid/assistierte Selbsttötung für Menschen mit schweren psychischen Störungen - Pro ${ }^{1}$
}

\author{
Assisted Suicide for People with Severe Mental Illness - Pro
}

\section{Pro}

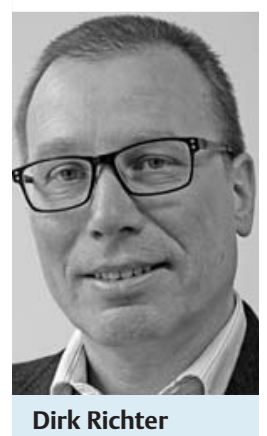

Die Debatte um Sterbehilfe hat in jüngster Zeit die Psychiatrie erreicht. In einigen europäischen Staaten, darunter auch in der Schweiz, kann aufgrund einer schweren psychischen Störung Assistenz beim selbstgewählten Tod in Anspruch genommen werden. In der Schweiz lag die Rate der Personen mit psychischen Störungen, welche die Assistenz genutzt haben, in den Jahren 2003-2008 bei 3,6\% aller Sterbewilligen [1]. Gesamthaft haben in den Niederlanden im Jahre 201556 Menschen mit psychischen Störungen diese Assistenz genutzt, total waren es mehr als 5000 Personen. Psychiatrisch relevant sind zusätzlich 109 Personen, die im Rahmen einer Demenz die Assistenz zur Lebensbeendigung in Anspruch genommen haben [2].

Selbstverständlich werfen diese Daten komplexe Fragen nach der ethischen $\mathrm{Zu}$ lässigkeit und nach psychiatrisch-professionellen Standards auf [3]. Besteht bei psychischen Erkrankungen ein ausreichendes Urteilsvermögen, das benötigt wird, um diesen schwerwiegenden Entscheid zu fällen? Sind lange Jahre schwersten psychischen Leidens tatsächlich ein ausreichender Grund für den zu erfüllenden Todeswunsch? Ließe sich trotz einer langen Leidensgeschichte nicht doch das psychische Leiden lindern? Sollten Todeswünsche von Menschen mit psychischen Störungen anders behandelt

\footnotetext{
${ }^{1}$ Frühere Versionen wurden präsentiert am World Forum der International Sociological Association, Research Committee Mental Health and Illness, Wien, Juli 2016, und bei der Akademischen Fachgesellschaft Psychiatrische Pflege Schweiz, Bern, September 2016. Ich danke den Teilnehmenden für die konstruktiven und herausfordernden Diskussionen.
}

werden als diejenigen von Menschen mit schweren oder terminalen körperlichen Erkrankungen?

Im Folgenden können diese Fragen nur kursorisch gestreift werden. Die Ausführungen konzentrieren sich auf psychiatrische Problemstellungen, die Terminologie bzw. Klassifikation, das Urteilsvermögen, die Diskriminierung von Menschen mit psychischen Störungen sowie die Einordnung der Problematik im Zusammenhang mit aktuellen sozialpsychiatrischen Konzepten.

Terminologie/Klassifikation: Psychiatrisches Lehrwissen beurteilt Todeswünsche in der Regel als Suizidalität und damit als ein Krankheitssymptom, zum Beispiel im Rahmen einer Depression. Dies hat eine Pathologisierung nahezu aller Todeswünsche zur Folge. Einer der am häufigsten zitierten Forschungsbefunde in diesem Zusammenhang ist, dass ca. 90\% aller Suizide im Zusammenhang mit psychischen Störungen geschehen. Diese komplette Pathologisierung wird in der Medizinund Bioethik sowie in den Rechtswissenschaften zunehmend infrage gestellt. Verschiedene Autorinnen und Autoren unterscheiden zwischen einem rationalen und aus freiem Willen gewählten Akt der Selbsttötung und einem Suizid im Rahmen einer psychischen Störung [4, 5]. Der Unterschied zwischen Selbsttötung und Suizid sollte gerade im Zusammenhang mit dem selbst gewählten Tod als Folge einer Krankheit beachtet werden [6].

Urteilsfähigkeit: Entgegen der Einschätzung vieler professionell Tätiger in der Psychiatrie ist die Urteilsfähigkeit im Zusammenhang mit der assistierten Selbsttötung in den meisten Fällen nicht derart beeinträchtigt, dass die Beihilfe zur Selbsttötung nicht möglich wäre. Eine aktuelle Studie über Ersuchen an Sterbehilfe-Organisationen im deutschsprachigen Raum hat ergeben, dass bei lediglich 8,7\% der Fälle, bei denen eine psychische Störung im Vordergrund stand, die Urteilsfähigkeit nicht gegeben war. Bei 52,4\% der Gesuche lag zwar eine psychopatholo- gische Beteiligung vor, die aber eine selbstbestimmte rationale Entscheidung nicht verhinderte. Und bei weiteren 38,8\% urteilte der/die Gutachtende, dass eine abgewogen rationale Entscheidung vorlag [7].

Diskriminierung von Menschen mit psychischen Störungen: Im Zusammenhang mit rationalen Selbsttötungen werden Menschen mit psychischen Störungen in verschiedener Hinsicht diskriminiert. So werden Sterbehilfeersuchen bei Menschen mit psychischen Störungen oftmals grundsätzlich abgelehnt. Des Weiteren wird psychischem Leiden in vielen Ländern eine geringere Relevanz als körperlichem Leiden zugesprochen oder aber es werden für psychische Leiden relativ einfache Lösungen propagiert. Schließlich kann es geschehen, dass trotz positiver gutachterlicher Stellungnahme das Ausstellen eines entsprechenden Rezepts für das notwendige Medikament verweigert wird - so geschehen in einem Fall in der Schweiz, der schließlich vor dem Europäischen Gerichtshof für Menschenrechte verhandelt wurde und zugunsten der klagenden Sterbewilligen entschieden wurde.

Dies alles widerspricht zwar nicht nationalen Gesetzen, aber dem Übereinkommen der Vereinten Nationen (UN-Behindertenrechtskonvention). Dort heißt es in Artikel 5, „(...) dass alle Menschen vor dem Gesetz gleich sind, vom Gesetz gleich zu behandeln sind und ohne Diskriminierung Anspruch auf gleichen Schutz durch das Gesetz und gleiche Vorteile durch das Gesetz haben." Und in Artikel 12 wird betont, „(...) dass Menschen mit Behinderungen in allen Lebensbereichen gleichberechtigt mit anderen Rechts- und Handlungsfähigkeit genießen." Wenn es um Selbsttötung Schwerstkranker geht, ist diese Gleichbehandlung in den meisten europäischen Staaten nicht garantiert. Selbsttötung und Sozialpsychiatrie: Aus guten und historisch nachvollziehbaren Gründen hat sich die deutschsprachige und insbesondere die deutsche Sozialpsy- 
chiatrie über lange Jahre zurückhaltend bis massiv ablehnend gegenüber assistierten Lebensbeendigungen verhalten. Differenzierte Betrachtungen sind hierzu erst in allerjüngster Zeit vorgelegt worden $[8,9]$. Unter der Perspektive aktueller sozialpsychiatrischer Konzepte wie Recovery, Empowerment und Risikoübernahme (positive risk-taking) sollte die lang gehegte Ablehnung jedoch revidiert werden. Es kann nicht angehen, dass Patientinnen und Patienten ermutigt werden, ihr Leben wieder in die eigenen Hände zu nehmen, für sie relevante Entscheidungen nicht mehr den Professionellen zu überlassen und Risiken einzugehen, die man ihnen zuvor nicht zugetraut hätte und dann aber im entscheidenden Moment die Gewährung dieses Rechts wieder zu entziehen. Empowerment heißt auch, das ultimative Risiko der assistierten Selbsttötung miteinzuschließen.

Schlussfolgerungen: Zusammenfassend kann festgestellt werden, dass es keine hinreichenden Gründe gibt, Menschen mit psychischen Störungen im Hinblick auf assistierte Selbsttötungen anders zu behandeln als Menschen mit primär körperlichen Erkrankungen. Folgt man der Unterscheidung von Suizid und Selbsttötung, dann kann letztere unter bestimmten Bedingungen auch für Menschen mit schweren psychischen Störungen nicht prinzipiell abgelehnt werden [10]. Zudem kann der vielfach befürchtete „Dammbruch“ angesichts der eingangs zitierten Daten nicht bestätigt werden. Es handelt sich um eine geringe Anzahl betroffener
Personen, welche die assistierte Lebensbeendigung in Anspruch nimmt. Der finale Paternalismus sollte somit der Vergangenheit angehören.

Sozialpsychiatrisches Handeln kann aber auch weitergehen: von der Unterstützung bei der Entscheidungsfindung bis hin zur Begleitung beim Sterben. Es gibt viele gute Gründe, Fachpersonen assistieren zu lassen [5]. Abgesehen davon, dass bekanntgewordene Selbsttötungsabsichten in den meisten Fällen missverstanden werden, zu automatischen Interventionen staatlicher und medizinischer Institutionen und unter Umständen zu Zwangseinweisungen führen, möchten viele Menschen nicht allein sterben. Zudem versprechen sich die betroffenen Personen eine sichere Durchführung und ein Sterben in Würde - all dies wäre bei der Alternative der allein und isoliert getätigten Lebensbeendigung nicht garantiert.

\section{Literatur}

1 Steck N, Junker C, Maessen $M$ et al. Suicide assisted by right-to-die associations: a population based cohort study. Int J Epidemiol 2014; 43: 614-622

2 Wilson C. Psychiatry's last taboo. New Scientist 2016; 231 (3083): 16-17

$3 \mathrm{Kim}$ SY, De Vries RG, Peteet JR. Euthanasia and Assisted Suicide of Patients With Psychiatric Disorders in the Netherlands 2011 to 2014. JAMA Psychiatry 2016; 73: $362-$ 368

4 den Hartogh G. Two Kinds of Suicide. Bioethics 2016: DOI: 10.1111/bioe.12287

5 Stefan S. Rational Suicide, Irrational Laws. Examining Current Approaches to Suicide in Policy and Law. New York: Oxford University Press; 2016

6 Gather J, Vollmann J. Suizidprävention und ärztlich assistierte Selbsttötung. Ein unauflösbarer ethischer Widerspruch für Psychiater? Nervenheilkunde 2015; 34: 430-434

7 Spittler JF. Urteilsfähigkeit, psychische Störung und Suizid-Beihilfe-Ansinnen. Schweiz Ärztezeitung 2016; 97: 435-437

8 Finzen A. Die neue Euthanasie in Belgien Wie steht es um die psychisch Kranken? Psychiat Prax 2015; 42: 411 -412

9 Gather J, Vollmann J. Die ärztlich assistierte Selbsttötung und advance care planning Medizinethische Überlegungen zur Selbstbestimmung am Lebensende von Menschen mit Demenz. Psychiat Prax 2014; 41: 385 391

10 Schuklenk U, van de Vathorst S. Treatmentresistant major depressive disorder and assisted dying. J Med Ethics 2015; 41: 577 583

Sie haben eine eigene Meinung zu diesem Thema? Dann schreiben Sie uns an: psychiat-praxis@thieme.de!

\section{Korrespondenzadresse}

\section{Dr. phil. habil. Dirk Richter}

Universitäre Psychiatrische Dienste Bern,

Direktion Psychiatrische Rehabilitation

Murtenstraße 46

3008 Bern, Schweiz

dirk.richter@puk.unibe.ch

Bibliografie

Dol http://dx.doi.org/

10.1055/s-0042-119789

Psychiat Prax 2016; 43: 411-412

(c) Georg Thieme Verlag KG

Stuttgart · New York

ISSN 0303-4259 